\title{
Common extracellular matrix regulation of myeloid cell activity in the bone marrow and tumor microenvironments
}

\author{
Sabina Sangaletti ${ }^{1} \cdot$ Claudia Chiodoni $^{1} \cdot$ Claudio Tripodo $^{2} \cdot$ Mario P. Colombo $^{1}$
}

Received: 21 November 2016 / Accepted: 6 May 2017 / Published online: 13 May 2017

(C) Springer-Verlag Berlin Heidelberg 2017

\begin{abstract}
The complex interaction between cells undergoing transformation and the various stromal and immunological cell components of the tumor microenvironment (TME) crucially influences cancer progression and diversification, as well as endowing clinical and prognostic significance. The immunosuppression characterizing the TME depends on the recruitment and activation of different cell types including regulatory $\mathrm{T}$ cells, myeloid-derived suppressor cells, and tumor-associated macrophages. Less considered is the non-cellular component of the TME. Here, we focus on the extracellular matrix (ECM) regulatory activities that, within the TME, actively contribute to many aspects of tumor progression, acting on both tumor and immune cells. Particularly, ECM-mediated regulation of tumor-associated immunosuppression occurs through the modulation of myeloid cell expansion, localization, and functional activities. Such regulation is not limited to
\end{abstract}

This paper is a Focussed Research Review based on a presentation given at the conference Regulatory Myeloid Suppressor Cells: From Basic Discovery to Therapeutic Application which was hosted by the Wistar Institute in Philadelphia, PA, USA, 16th-19th June, 2016. It is part of a Cancer Immunology, Immunotherapy series of Focussed Research Reviews.

Sabina Sangaletti and Claudia Chiodoni have equally contributed to this work.

Mario P. Colombo

mariopaolo.colombo@istitutotumori.mi.it

1 Molecular Immunology Unit, Department of Experimental Oncology and Molecular Medicine, Fondazione IRCCS Istituto Nazionale dei Tumori, Via Amadeo, 42, 20133 Milan, Italy

2 Tumor Immunology Unit, University of Palermo, Palermo, Italy the TME but occurs also within the bone marrow, wherein matricellular proteins contribute to the maintenance of specialized hematopoietic stem cell niches thereby regulating their homeostasis as well as the generation and expansion of myeloid cells under both physiological and pathological conditions. Highlighting the commonalities among ECM-myeloid cell interactions in bone marrow and TME, in this review we present a picture in which myeloid cells might sense and respond to ECM modifications, providing different ECM-myeloid cell interfaces that may be useful to define prognostic groups and to tailor therapeutic interventions.

Keywords Regulatory myeloid suppressor cells . SPARC - Tumor microenvironment - Bone marrow niche · Extracellular matrix

$\begin{array}{ll}\text { Abbreviations } \\ \text { AML } & \text { Acute myeloid leukemia } \\ \text { BM } & \text { Bone marrow } \\ \text { DLBCL } & \text { Diffuse large B cell lymphoma } \\ \text { ECM } & \text { Extracellular matrix } \\ \text { EMT } & \text { Epithelial to mesenchymal transition } \\ \text { HSC } & \text { Hematopoietic stem cell } \\ \text { IFN } \gamma & \text { Interferon gamma } \\ \text { IL } & \text { Interleukin } \\ \text { LAIR } & \text { Leukocyte-associated Ig-like receptor } \\ \text { LOX } & \text { Lysyl-oxidase } \\ \text { MDS } & \text { Myelodisplastic syndrome } \\ \text { MDSC } & \text { Myeloid-derived suppressor cell } \\ \text { NETs } & \text { Neutrophil extracellular traps } \\ \text { OPN } & \text { Osteopontin } \\ \text { PMN } & \text { Polymorphonuclear } \\ \text { RNS } & \text { Reactive nitrogen species } \\ \text { ROS } & \text { Reactive oxygen species }\end{array}$


TAM Tumor-associated macrophage

TME Tumor microenvironment

TNF Tumor necrosis factor

TSP-1 Thrombospondin-1

\section{Relevance of the extracellular matrix in tumors}

Although oncogene activation and oncosuppressor gene inactivation act as drivers of cancer development, fullblown tumorigenesis and malignant progression require the acquisition of several additional capabilities that enable tumor cells to survive, proliferate, invade, metastasize, and evade immune recognition and destruction [1]. Furthermore, hematopoietic cells and stromal cells are involved in all phases of carcinogenesis and cancer progression and, in turn, are themselves modified by the developing cancer. The comparison between normal and transformed tissues shows a progressive enrichment in inflammatory cells associated with variable changes in the stromal composition determining the so-called "desmoplastic reaction" that, in some cases, configures marked fibrosis characterized by excessive extracellular matrix (ECM) deposition as seen in some breast cancer histotypes [2].

Within the tumor microenvironment (TME), cancer cells and activated fibroblasts in concert with some immune cells, such as macrophages, comprise the main source of ECM molecules that interpose between the tumor and immune cells and can favor or impede their cross-communication [3-5]. In addition to its expected scaffold properties, ECM exerts regulatory functions on both tumor and stromal cells, suggesting that a comprehensive understanding of the reciprocal cross-talk between the ECM, tumor cells, and stromal cells might impact the success of cancer therapy programs [6]. For example, collagen deposition and stiffness up-regulate integrin signaling, which eventually promotes tumor cell survival and proliferation. The over-production of lysyl-oxidase (LOX), an enzyme that cross-links collagen fibers, promotes extracellular signalregulated kinase assembly and phosphoinositide 3 kinase signaling, facilitating oncogenic transformation [7].

The key role of ECM in cancer progression is strengthened by its prognostic relevance, such as in the case of high-grade breast cancers where the ECM-gene profile classification correlates with clinical outcome and response to therapy $[8,9]$. A signature termed ECM3, characterized by up-regulation of SPARC, COL1A1, COL5A2, LAMA4, COL6A3, and MMP11 genes, delineates the most robust cluster with stable tumor partition within 6 independent datasets of more than 600 samples, allowing the re-classification of breast cancers into two groups of ECM3 or nonECM3 type [9]. The ECM3 signature characterizes approximately $35 \%$ of breast carcinomas including high-grade cases, which undergo epithelial to mesenchymal transition (EMT) and respond poorly to therapy [10]. Notably, the prognostic value of this signature is conserved among all breast cancer molecular subtypes suggesting its relevance in providing information related to the biology of these tumors beyond cancer cell-intrinsic characteristics.

A similar ECM gene cluster, comprising COL1A1, FN1, LOX, SPARC, TIMP3, and COLIA2 has been associated with the resistance to tamoxifen when administered as a first-line therapy inpatients with metastatic breast cancer [11]. More recently, a SPARC-driven gene expression signature obtained from murine models predicted the clinical outcome of patients with HER2-enriched breast cancer subtypes [12]. Notably, signatures enriched in collagen genes together with $L O X, T H B S 2, T I M P 3$, and SPARC have also been associated with poor survival after adjuvant chemotherapy and with a high hazard of death in ovarian cancer $[13,14]$. Similarly, in colon cancer, Gene Set Enrichment Analysis (GSEA) identified subsets of ECM-encoding genes that were strongly correlated with the metastatic potential of primary cancers [15].

Other non-epithelial tumors for which a poor clinical outcome has been associated with the presence of ECM clusters are Hodgkin's lymphoma (genes: COL18A1, COL6A1, MMP2, MMP3, and TIMP1) and some types of diffuse large B cell lymphomas (DLBCLs). In DLBCL, an ECM-related gene signature fronted by SPARC has been reported to be over expressed in cases with a favorable outcome [16]. Notably, in CD5+ DLBCL, a subtype of DLBCL with an aggressive disease course, the ECMrelated genes POSTN, SPARC, COL1A1, COL3A1, CSTK, $M M P 9$, and $L A M B 3$ were down-regulated [17], suggesting that modification leading to either up-or down-regulation of ECM genes may have a role in shaping the biology of both solid and hematologic malignancies. In this context, we have recently reported that the absence of Sparc in autoimmunity-prone Fas-mutant mice was instrumental to promote the transformation of CD5+ B-cells toward a malignant lymphoproliferative disease [18].

\section{Interaction between the ECM and the immune system}

The immune system is the most relevant component of the TME governing host-tumor interactions. Active immunosuppression depends on the recruitment and activation of different cell types including regulatory $\mathrm{T}$ cells, myeloidderived suppressor cells (MDSCs), and tumor-associated macrophages. Similarly to what has been described for the ECM, it is possible to predict the clinical outcome of a tumor based on the presence and localization of different $T$ lymphocyte subsets [19] or on the expression of 
immune cell-related gene signatures. Such signatures can be identified using tools such as CIBERSORT, a computational method for inferring leukocyte representation in bulk tumors [20]. In particular, a recent application of CIBERSORT utilized the expression profile of 22 distinct leukocyte subsets as "barcodes", capable of distinguishing different immune cell types, to screen the gene expression profiles of 18,000 cancer patients of different tumor types, for whom survival data were available [21]. This analysis showed that enrichment in $\mathrm{T}$ cells, gamma-delta $\mathrm{T}$ cells, and plasma cells was associated with increased survival in all 39 types of cancer analyzed. In contrast, a polymorphonuclear (PMN) leukocyte signature was associated with the worst survival in all the histotypes analyzed [21]. Notably, as for the ECM signatures, the immune profiles were also prognostic across different tumors independently from their molecular subtypes, again supporting the idea that such signatures are indicative of common biological features conserved across different histotypes. Identification of the key molecular mechanisms responsible for such common biology is, therefore, likely to open to new therapeutic possibilities that function independently from tumor histotypes and molecular subtypes.

In addition, although the ECM signature or the immune signature may be independently prognostic, per se, their intersection might be even more informative for patient selection toward the most appropriate therapeutic regimen. In this regard a new method has been described to estimate, by gene expression, the tumor infiltration of fibroblasts and endothelial cells along with the immune cells, thus offering a compelling microenvironment-based patient stratification system associated with disease outcome [22]. We also observed differential enrichment in immune-related genes between non-ECM3 and ECM3 clusters during the process of studying the biological mechanism influencing the interaction between ECM3 signature and tumor grade. In particular, high-grade ECM3 and non-ECM3 tumors differed in the expression of genes related to immune cell functions, with ECM3 tumors deficient for NK, T, and B cell-related genes otherwise enriched in their non-ECM3, less aggressive counterparts. Conversely, ECM3 tumors were enriched in myeloid cells that, notably, were also localized in close contact with tumor cells.

The reduced number of T cells in ECM3 + tumors might be explained in several ways. The first points to the role of collagen in coordinating $\mathrm{T}$ cell migration and activation. Collagen fibers can dictate the migratory trajectory of $\mathrm{T}$ cells and are necessary to stabilize immunological synapses whereas an excessively tight ECM negatively affects T cell infiltration into the tumor [23]. Another possible explanation for under-represented T cells within ECM3+ tumors might depend on the enrichment in immunosuppressive myeloid cells [10]. Tumor-associated myeloid cells constitute the main negative regulators of antitumor immune responses at the tumor site. A key characteristic of these cells is their generation of reactive oxygen (ROS) and reactive nitrogen species (RNS) in the tumor microenvironment. ROS and RNS promote post-translational modification of chemokines that, as consequence, loose their chemotactic activity on $T$ cells [24]. In particular, chemokines normally present in the tumor microenvironment including CXC12, CCL2, or CCL5 can reduce their binding activity to the corresponding receptor on $\mathrm{T}$ cells when exposed to ROS/RNS-induced post-translational modification [25]. For example, the reduced binding affinity of nitrated/nitrosylated CCL2 to its receptor CCR2 impairs the homing of CD8+ T lymphocytes [25]. Conversely, the nitrated/nitrosylated CCL2 does not lose the ability to recruit MDSCs to the tumor site, likely owing to a higher expression of CCR2 on myeloid cells, which may explain the peculiar enrichment in myeloid cells at the expense of $\mathrm{T}$ cells that occurs in ECM3 + tumors.

Furthermore, defective collagen assembly in Sparc-deficient mice is associated with over-production of myeloid cells and/or changes in their activation status in response to different stimuli. Indeed, Sparc-deficient mice show an increased onset of autoimmune conditions (systemic lupus erythematosus) in response to chemically induced (unpublished data) or Fas-driven [18] stimulation through a mechanism involving deregulated neutrophil production and activation [26].

Overall, these pieces of evidence together suggest that myeloid cells may not only serve as a primary sensor of ECM modifications but also that they may be relevant for the aggressiveness of ECM-rich tumors and, therefore, might be targeted for therapeutic purposes. In this context, we provided data showing that the EMT features characteristic of ECM3 + tumors can be reverted using immunomodulatory compounds acting on myeloid cells. Notably, the reversion of the EMT phenotype was associated with increased tumor cell proliferation and, therefore, with a higher sensitivity to DNA-intercalating drugs, such as doxorubicin [10].

\section{Myeloid cell-ECM interactions}

We envisage two possible ways for ECM to interfere with myeloid cell behavior: acting on the recruitment of myeloid cells, and impacting their activation and/or function. As an example, in 4T1 murine mammary carcinoma, the presence of an ECM gradient associated with the enrichment of mesenchymal traits determines a redistribution of myeloid cells that concentrate mainly in the collagen-rich mesenchymal invasive areas (our unpublished observation). During cancer development the intense stromal remodeling recruits 
macrophages, whose physiological "wound healing" function is instead converted into pro-tumorigenic activity. In mice lacking SPARC, the decreased collagen deposition allows increased infiltration of M1 macrophages that, endowed with interferon gamma (IFN $\gamma$ ) and tumor necrosis factor (TNF) production, exhibit anti-tumor properties [4]. This suggests that modifications in the ECM may simultaneously influence the number and function of immune cells within the tumor microenvironment. Consistent with this evidence, collagen type I can either promote tumor infiltration by immune cells or inhibit macrophage anti-tumor activity by blocking M1 polarization and activation [27].

The inhibitory activity of collagens on immune cells is likely mediated by specific surface receptors, prototypically the leukocyte-associated Ig-like receptor (LAIR) that is expressed on the surface of most immune cells [28] to convey regulatory signals upon collagen binding, an event that could contribute to tumor evasion from immunosurveillance. We analyzed the expression of LAIR-1 in previously generated datasets of human immune cells and found that LAIR-1 is expressed by almost all leukocytes, albeit at different levels. In particular, the expression pattern of LAIR-1 was inversely associated on PMNs along with their differentiation status: from higher expression on common myeloid progenitors, granulocyte-monocytes progenitors, pro-myelocytes, and myelocytes to lower or absent expression on PMNs in the bone marrow (BM) or in the peripheral blood. Its expression, however, could be re-induced in mature PMNs from peripheral tissues upon stimulation with inflammatory compounds such as phorbol-12-myristate-13-acetate (PMA) or lipopolysaccharide ([28] and Sangaletti unpublished).

These observations suggest that ECM modification might impact the homeostasis of compartments designated toward myeloid cell generation such as the BM and that ECM changes might constitute part of the initiation of diseases associated with the loss of quiescence or with the abnormal outgrowth of immature myeloid cells, such as myelodisplastic syndrome (MDS) and other hematological disorders. Additionally, an expansion of immature myeloid cells such as detectable in the peripheral blood of patients carrying solid tumors might occur in response to similar ECM modifications. These expanded myeloid cells exhibit the features of promyelocytic cells and are responsible for the immune suppression occurring in patients with breast and colorectal cancer in correlation with disease progression [29]. At present, however, it is still not understood whether tumor-expanded myeloid cells, such as physiologic promyelocytes, retain a profile of LAIR-1 expression conducive for response to collagen regulation. Similarly, it is not known whether collagens are involved in maintaining tumor-associated myeloid cell immunosuppressive phenotype, although in the presence of inflammatory stimuli (i.e.,
IFN- $\gamma$, TNF) that would be expected to mediate myeloid cell activation [30]. Some data on the possible role of collagens in repressing PMN activation have been obtained from studying neutrophil extracellular traps (NETs) in the context of autoimmunity, wherein IFN- $\gamma$ and C5a induction of NETs could be inhibited by seeding stimulated PMN onto a collagen matrix. Notably, such collagen inhibitory effect is prevented in PMNs transiently silenced for LAIR-1 expression [18].

\section{ECM-regulation of MDSCs in cancer: a SPARC experience}

As human high grade ECM3+ breast cancers are characterized by high SPARC expression, enriched myeloid cell infiltration, and paucity of $\mathrm{T}$ cells, we investigated whether SPARC has any functional relation with MDSC recruitment and suppressive activities. This was achieved by over expressing SPARC in Sparc-deficient or lowexpressing cancer cells. Sparc-transduction in mammary tumor cells promoted MDSC expansion, especially of the granulocytic subset (ratio 10:1 with the monocytic subset). Myeloid cells expanded by tumors over expressing SPARC were functionally different from those expanded by Sparc-deficient tumors. The former were more efficient in suppressing T cell proliferation and showed higher Stat3, interleukin (IL)-6, arginase I, and COX-2 expression. This phenotype was reliant upon SPARC-induced Cox-2-regulation of GM-CSF and IL-6 production, two master cytokines involved in MDSC differentiation [31]. Consistent with this observation, shRNA-mediated SPARC knockdown markedly reduced primary tumor growth and completely abolished lung colonization of murine $4 \mathrm{~T} 1$ cells by affecting cell cycle and COX-2-mediated expansion of MDSCs [12]. The activation of COX-2 in a SPARC-rich microenvironment was related to the higher fibrillar collagen deposition, as collagen represents a direct inducer of COX-2 [32] (Fig. 1). The relevance of the collagen/COX-2 axis in mammary tumorigenesis has been clearly shown in mouse models investigating the link between pregnancy and ductal carcinoma in situ (DCIS) progression [32]. Accordingly, PyMT/Colla $1^{\mathrm{tm} l \mathrm{ja} e}$ mice, which carry a mutation in the collagenase cleavage site of collagen 1a1, developed tumors characterized by dense collagen deposition and associated with increased inflammation in comparison to PyMT tumors from wild-type mice. COX-2 over expression was responsible for the robust inflammatory milieu of collagen-dense tumors and led to the recruitment of tumorassociated macrophages (TAMs) and tumor-associated neutrophils; a setting that could be reverted by the use of celecoxib, a selective COX-2 inhibitor [33]. In addition, the 
Fig. 1 Matricellular protein regulation of tumor-associated myelopoiesis. Within the TME matricellular proteins have been shown to regulate myeloid cell behavior and functions at different levels. Tumorproduced SPARC and OPN promote MDSC expansion in $\mathrm{BM}$ and spleen via production of VEGF, IL-6, and GM-CSF $[10,49]$. Tumor-derived SPARC promotes the recruitment of MDSCs at the tumor site via COX-2 and CXCR-4/CXCL12 axes, where they promote EMT and metastases [10]. OPN sustains the recruitment of MDSCs at the lung metastatic site where they are instrumental for the instruction of the metastatic niche. In addition, periostin takes part in creating the premetastatic niche [47]. Conversely, Gr-1-derived TSP-1 has been shown to counteract metastases development

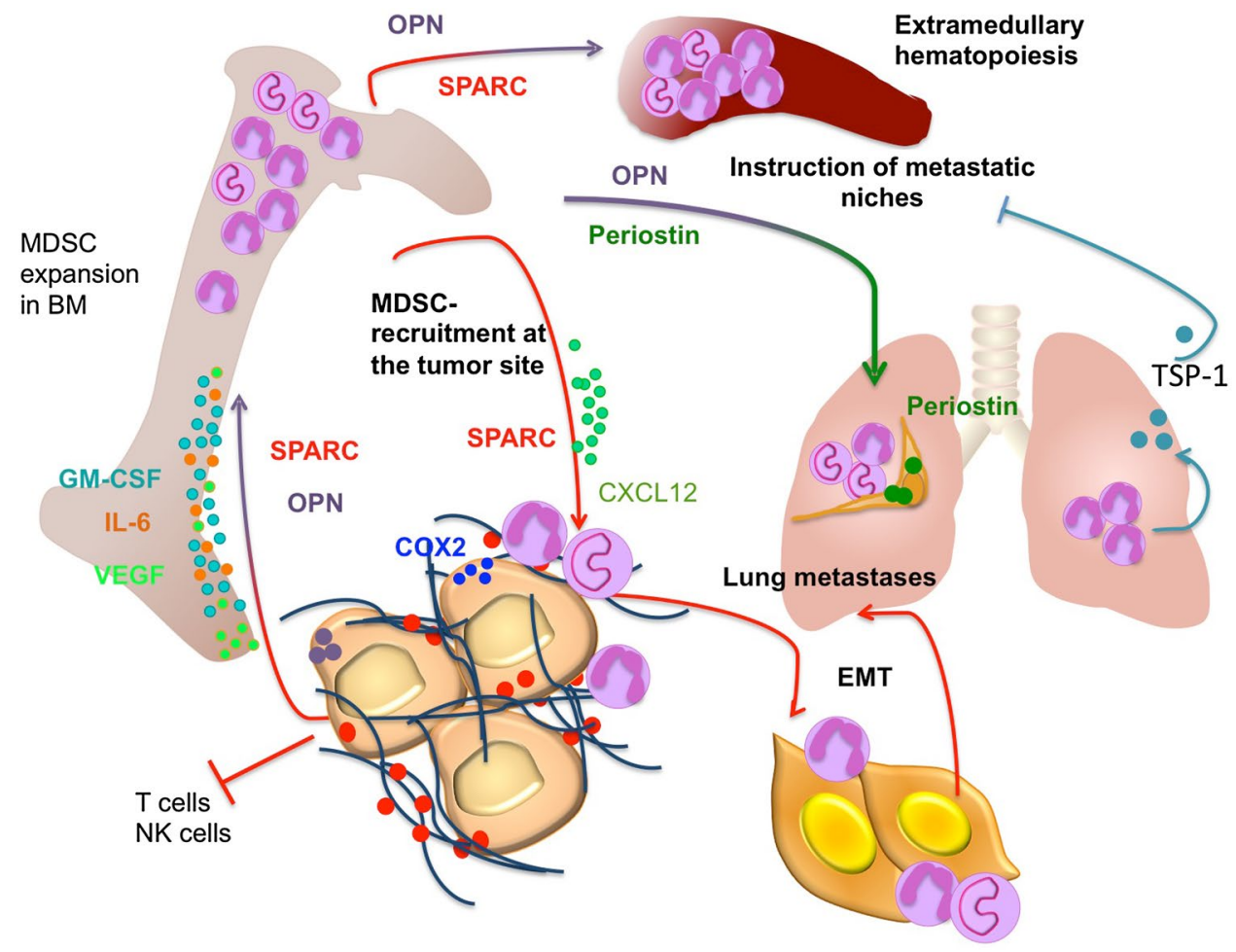

use of celecoxibin our SPARC-hyper-expressing mammary tumors reduced MDSC recruitment at the tumor site [10].

Furthermore, the cross-talk between myeloid cells and collagens has been elegantly shown in models of skin fibrosis in which myeloid-cell-restricted IL-4R $\alpha$ signaling controls collagen fibril assembly. After mechanical skin injury, IL-4R $\alpha$ signaling, in response to IL-4 and IL-13, coordinates the timely switch of macrophages from the inflammatory to the resolution phase of wound repair, also controlling the architecture of collagen fibrils and the biochemistry of collagen cross-linking [34]. Myeloid-restricted IL-4R $\alpha$ deficiency has been associated with defective granulation tissue formation and wound repair. Similarly, Sparc-deficient mice are characterized by defective wound repair [35] where as Sparc-competent M2 macrophages produce collagen and sustain stromatogenesis and metastasis [4, 5].

Overall, these data suggest that SPARC, collagen, COX2, and myeloid cells (TAMs and MDSCs) are entwined in the tumor microenvironment, where they simultaneously support ECM fiber deposition and the instruction of an immunosuppressive environment.

\section{Matricellular protein regulation of myelopoiesis}

The ECM protein SPARC belongs to the class of matricellular proteins, a group of molecules that exert regulatory rather than structural roles within the ECM. Other matricellular proteins are thrombospondin 1 and 2, CCN family proteins, periostin, osteopontin (OPN), and tenascins. Matricellular proteins share some common features including the capacity to interact with ECM structural components (i.e., fibrillar collagens), growth factors, and proteases and thereby the capacity to interfere with physiological processes such as wound healing and repair [36]. Increasing evidence suggests that matricellular proteins also share the capacity of regulating myeloid cell behavior including their production, recruitment, and activation. In particular, these proteins can contribute to the establishment of BM specialized niches regulating the maintenance, proliferation, quiescence, and homing of hematopoietic stem cells (HSCs) (Fig. 2; Table 1). Different matricellular proteins have been described to take part in BM HSC niches, where they are provided by osteoblasts or BM mesenchymal stem cells and subsequently stimulate or inhibit the proliferation of HSCs. In this context, SPARC deficiency affects HSC function under certain stress conditions, providing survival benefit to SPARC-deficient mice treated repeatedly with the chemotherapeutic agent 5-FU [37]. This effect was clearly niche-dependent rather than cell autonomous. Consistent with this concept we showed that SPARC produced by BM stromal cells regulates myeloid cell production [38] and B lymphopoiesis [39]. Moreover, we reported an enhanced reactive myeloproliferative response to thrombopoietin in the absence of stromal SPARC or from the transplantation of defective Apc ${ }^{\text {min }}$ mutant hematopoietic cells into Sparc $^{-/-}$but not wild-type recipient BM stroma [38]. Another matricellular protein 

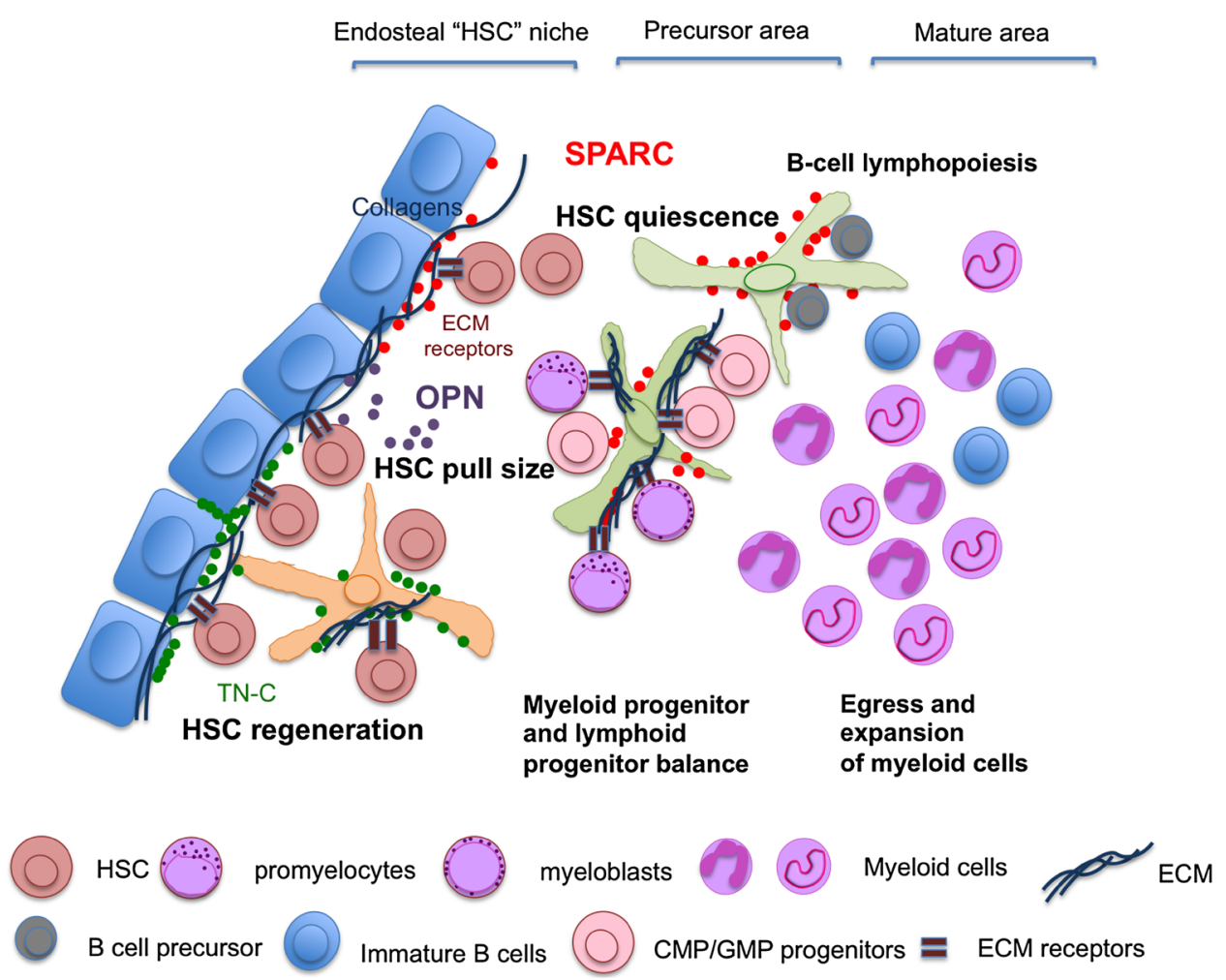

Fig. 2 Matricellular protein regulation of the BM niche. Within the BM, HSCs and progenitors (CMPs, GMPs) and immature myeloid cells (myeloblasts and promyelocytes) express the ECM sensing receptor LAIR-1, suggesting that ECM modification might impact the homeostasis of compartments delegated to myeloid cell generation in the BM. Through their capacity to interact with ECM structural components (fibrillar collagens), or through a direct effect on HSCs, matricellular proteins, which are mainly produced by BM mesenchymal elements, share the capacity to regulate hematopoietic and myeloid cell behavior in the BM microenvironment. In such a context SPARC, a collagen regulator, has been shown to be involved in con-

that has been described to be a key component of the HSC niche and a regulator of primitive hematopoietic progenitor cells is OPN, which is produced by osteoblasts, acts as a negative regulator of hematopoiesis, and contributes to HSC localization [40].

The production SPARC and OPN is often dysregulated in hematopoietic malignancies characterized by the altered production of hematopoietic and myeloid cells, such as chronic myeloid leukemia (CML) [41], MDS [42] and acute myeloid leukemia (AML) [43, 44]. Similarly, Tenascin-C is also a critical component of the $\mathrm{BM}$ microenvironment. In a setting of BM transplantation, Tenascin-C supports the engraftment of donor BM [45] (Fig. 2). Therefore, it appears that a deregulated production of myeloid cells at sites in which these cells are generated is often associated with the altered presence of matricellular proteins, suggesting that these proteins may be also involved in regulating extramedullary trolling HSC quiescence, myeloid cell proliferation, and B cell lymphopoiesis. Accordingly, the absence of SPARC was associated with the rapid return to quiescence of HSCs in BM transplantation settings, increased myelopoiesis in response to myeloproliferative spurs, and to inefficient B cell lymphopoiesis. OPN is another key component of the HSC niche; a product of osteoblasts, it acts as negative regulator of hematopoiesis and takes part in HSC localization. Similarly to SPARC and OPN, Tenascin-C is a critical component of the $\mathrm{BM}$ microenvironment. In a setting of BM transplantation, Tenascin$\mathrm{C}$ supports the engraftment of donor BM cells

myelopoiesis, which is characterized by the accumulation of peripheral MDSCs in secondary lymphoid organs of tumor-bearing mice. Additionally, OPN has been shown to facilitate the accumulation of splenic MDSCs in mice bearing colon cancer by accelerating the differentiation of HSCs and heterogeneous myeloid progenitor cells [46]. Accordingly, OPN re-expression in OPN-deficient breast cancer cells promotes MDSC increase in the peripheral blood, spleen, and tumor (Chiodoni unpublished). Similarly, SPARC-expression in breast cancer cells promoted myelopoiesis, leading to the expansion of BM, splenic, and circulating MDSCs. Notably, matricellular proteins, particularly periostin and OPN, also contribute to the organization of the MDSC-driven pro-metastatic lung niche, albeit with different mechanisms [47-49]. Such mechanisms utilize the lectin-type oxidized LDL receptor-1 (LOX-1), which notably identifies human MDSCs in cancer patients [50]. In contrast to periostin and OPN, 
Tsp- 1 produced by $\mathrm{Gr}-1^{+}$myeloid cells contributes to the generation of a metastasis-resistant microenvironment [51].

\section{Concluding remarks}

The imbalance of the tumor immune microenvironment in favor of an immunosuppressive milieu rather than toward immune activation is generally ascribed to the activity of soluble mediators, such as chemokines, cytokines, growth factors, and ROS that regulate the recruitment, phenotype, and function of immune cells. However, increasing evidence points to the ECM as an active contributor in molding the tumor-associated immunosuppressive microenvironment and denotes a role for non-structural matricellular proteins in regulating stromal remodeling and immune cell recruitment and function in tumors. In this context, myeloid cells may be viewed as major sensors of ECM modifications. The parallel finding of ECM-rich and myeloid cell-rich gene signatures endorsing independent prognostic significance in breast cancer, irrespective of the histotype or molecular subtype, opens new prospects related to targeting the functional ECM-myeloid cell interface for the development of new effective therapeutic agents or strategies.

\section{Compliance with ethical standards}

Conflict of interest The authors have no conflict of interest to declare.

Funding This work was supported by Associazione Italiana per la Ricerca sul Cancro (IG 10137 to Mario P. Colombo, MFAG 12810 to Sabina Sangaletti, IG 17261 to Claudia Chiodoni), and the Italian Ministry of Health (GR-2013-02355637 to Sabina Sangaletti).

\section{References}

1. Hanahan D, Weinberg RA (2011) Hallmarks of cancer: the next generation. Cell 144:646-674. doi:10.1016/j.cell.2011.02.013

2. Acerbi I, Cassereau L, Dean I et al (2015) Human breast cancer invasion and aggression correlates with ECM stiffening and immune cell infiltration. Integr Biol (Camb) 7:1120-1134. doi:10.1039/c5ib00040h

3. Afik R, Zigmond E, Vugman M et al (2016) Tumor macrophages are pivotal constructors of tumor collagenous matrix. J Exp Med 213:2315-2331. doi:10.1084/jem.20151193

4. Sangaletti S, Stoppacciaro A, Guiducci C, Torrisi MR, Colombo MP (2003) Leukocyte, rather than tumor-produced SPARC, determines stroma and collagen type IV deposition in mammary carcinoma. J Exp Med 198:1475-1485. doi:10.1084/ jem.20030202

5. Sangaletti S, Di Carlo E, Gariboldi S et al (2008) Macrophagederived SPARC bridges tumor cell-extracellular matrix interactions toward metastasis. Cancer Res 68:9050-9059. doi:10.1158/0008-5472.CAN-08-1327 
6. Pickup MW, Mouw JK, Weaver VM (2014) The extracellular matrix modulates the hallmarks of cancer. EMBO Rep 15:1243-1253. doi:10.15252/embr.201439246

7. Levental KR, Yu HM, Kass L et al (2009) Matrix crosslinking forces tumor progression by enhancing integrin signaling. Cell 139:891-906. doi:10.1016/j.cell.2009.10.027

8. Bergamaschi A, Tagliabue E, Sørlie T et al (2008) Extracellular matrix signature identifies breast cancer subgroups with different clinical outcome. J Pathol 214:357-367. doi:10.1002/ path. 2278

9. Triulzi T, Casalini P, Sandri M et al (2013) Neoplastic and stromal cells contribute to an extracellular matrix gene expression profile defining a breast cancer subtype likely to progress. PLoS One 8:e56761. doi:10.1371/journal.pone.0056761

10. Sangaletti S, Tripodo C, Santangelo A et al (2016) Mesenchymal transition of high-grade breast carcinomas depends on extracellular matrix control of myeloid suppressor cell activity. Cell Rep 17:233-248. doi:10.1016/j.celrep.2016.08.075

11. Helleman J, Jansen MP, Ruigrok-Ritstier K et al (2008) Association of an extracellular matrix gene cluster with breast cancer prognosis and endocrine therapy response. Clin Cancer Res 14:5555-5564. doi:10.1158/1078-0432.Ccr-08-0555

12. Guttlein LN, Benedetti LG, Fresno C et al (2017) Predictive outcomes for HER2-enriched cancer using growth and metastasis signatures driven by SPARC. Mol Cancer Res 15:304-316. doi:10.1158/1541-7786.MCR-16-0243-T

13. Cheon DJ, Tong YG, Sim MS et al (2014) A collagen-remodeling gene signature regulated by TGF-beta signaling is associated with metastasis and poor survival in serous ovarian cancer. Clin Cancer Res 20:711-723. doi:10.1158/1078-0432.Ccr-13-1256

14. Zhang W, Ota T, Shridhar V, Chien J, Wu BL, Kuang R (2013) Network-based survival analysis reveals subnetwork signatures for predicting outcomes of ovarian cancer treatment. PLoS Comput Biol 9:e1002975. doi:10.1371/journal.pcbi.1002975

15. Naba A, Clauser KR, Whittaker CA, Carr SA, Tanabe KK, Hynes RO (2014) Extracellular matrix signatures of human primary metastatic colon cancers and their metastases to liver. BMC Cancer 14:518. doi:10.1186/1471-2407-14-518

16. Lenz G, Wright G, Dave SS et al (2008) Stromal gene signatures in large-B-cell lymphomas. N Engl J Med 359:2313-2323. doi:10.1056/NEJMoa0802885

17. Jain P, Fayad LE, Rosenwald A, Young KH, O'Brien S (2013) Recent advances in de novo CD5(+) diffuse large B cell lymphoma. Am J Hematol 88:798-802. doi:10.1002/ajh.23467

18. Sangaletti S, Tripodo C, Vitali C et al (2014) Defective stromal remodeling and neutrophil extracellular traps in lymphoid tissues favor the transition from autoimmunity to lymphoma. Cancer Discov 4:110-129. doi:10.1158/2159-8290.Cd-13-0276

19. Galon J, Costes A, Sanchez-Cabo F et al (2006) Type, density, and location of immune cells within human colorectal tumors predict clinical outcome. Science 313:1960-1964. doi:10.1126/ science.1129139

20. Newman AM, Liu CL, Green MR et al (2015) Robust enumeration of cell subsets from tissue expression profiles. Nat Methods 12:453-457. doi:10.1038/nmeth.3337

21. Gentles AJ, Newman AM, Liu CL et al (2015) The prognostic landscape of genes and infiltrating immune cells across human cancers. Nat Med 21:938-945. doi:10.1038/nm.3909

22. Becht E, Giraldo NA, Lacroix L et al (2016) Estimating the population abundance of tissue-infiltrating immune and stromal cell populations using gene expression. Genome Biol 17:218. doi:10.1186/s13059-016-1070-5

23. Salmon H, Franciszkiewicz K, Damotte D et al (2012) Matrix architecture defines the preferential localization and migration of $\mathrm{T}$ cells into the stroma of human lung tumors. J Clin Invest 122:899-910. doi:10.1172/Jci45817
24. Lu TY, Gabrilovich DI (2012) Molecular pathways: tumor-infiltrating myeloid cells and reactive oxygen species in regulation of tumor microenvironment. Clin Cancer Res 18:4877-4882. doi:10.1158/1078-0432.CCR-11-2939

25. De Sanctis F, Sandri S, Ferrarini G et al (2014) The emerging immunological role of post-translational modifications by reactive nitrogen species in cancer microenvironment. Front Immunol 5:69. doi:10.3389/fimmu.2014.00069

26. Smith CK, Kaplan MJ (2015) The role of neutrophils in the pathogenesis of systemic lupus erythematosus. Curr Opin Rheumatol 27:448-453. doi:10.1097/Bor.0000000000000197

27. Kaplan G (1983) In vitro differentiation of human monocytes. Monocytes cultured on glass are cytotoxic to tumor cells but monocytes cultured on collagen are not. J Exp Med 157:2061-2072

28. Lebbink RJ, de Ruiter T, Adelmeijer J et al (2006) Collagens are functional, high affinity ligands for the inhibitory immune receptor LAIR-1. J Exp Med 203:1419-1425. doi:10.1084/ jem. 20052554

29. Solito S, Falisi E, Diaz-Montero CM et al (2011) A human promyelocytic-like population is responsible for the immune suppression mediated by myeloid-derived suppressor cells. Blood 118:2254-2265. doi:10.1182/blood-2010-12-325753

30. Mantovani A, Cassatella MA, Costantini C, Jaillon S (2011) Neutrophils in the activation and regulation of innate and adaptive immunity. Nat Rev Immunol 11:519-531. doi:10.1038/ nri3024

31. Marigo I, Bosio E, Solito S et al (2010) Tumor-induced tolerance and immune suppression depend on the C/EBPbeta transcription factor. Immunity 32:790-802. doi:10.1016/j. immuni.2010.05.010

32. Lyons TR, O'Brien J, Borges VF et al (2011) Postpartum mammary gland involution drives progression of ductal carcinoma in situ through collagen and COX-2. Nat Med 17:1109-1115. doi: $10.1038 / \mathrm{nm} .2416$

33. Esbona K, Inman D, Saha S, Jeffery J, Schedin P, Wilke L, Keely P (2016) COX-2 modulates mammary tumor progression in response to collagen density. Breast Cancer Res 18:35. doi:10.1186/s13058-016-0695-3

34. Knipper JA, Willenborg S, Brinckmann J et al (2015) Interleukin-4 receptor alpha signaling in myeloid cells controls collagen fibril assembly in skin repair. Immunity 43:803-816. doi:10.1016/j.immuni.2015.09.005

35. Basu A, Kligman LH, Samulewicz SJ, Howe CC (2001) Impaired wound healing in mice deficient in a matricellular protein SPARC (osteonectin, BM-40). BMC Cell Biol 2:15. doi:10.1186/1471-2121-2-15

36. Murphy-Ullrich JE, Sage EH (2014) Revisiting the matricellular concept. Matrix Biol 37:1-14. doi:10.1016/j.matbio.2014.07.005

37. Ehninger A, Boch T, Medyouf H, Müdder K, Orend G, Trumpp A (2014) Loss of SPARC protects hematopoietic stem cells from chemotherapy toxicity by accelerating their return to quiescence. Blood 123:4054-4063. doi:10.1182/blood-2013-10-533711

38. Tripodo C, Sangaletti S, Guarnotta C et al (2012) Stromal SPARC contributes to the detrimental fibrotic changes associated with myeloproliferation whereas its deficiency favors myeloid cell expansion. Blood 120:3541-3554. doi:10.1182/ blood-2011-12-398537

39. Sangaletti S, Tripodo C, Portararo P et al (2014) Stromal niche communalities underscore the contribution of the matricellular protein SPARC to B-cell development and lymphoid malignancies. Oncoimmunology 3:e28989. doi:10.4161/onci.28989

40. Nilsson SK, Johnston HM, Whitty GA et al (2005) Osteopontin, a key component of the hematopoietic stem cell niche and regulator of primitive hematopoietic progenitor cells. Blood 106:1232-1239. doi:10.1182/blood-2004-11-4422 
41. Giallongo C, La Cava P, Tibullo D et al (2013) SPARC expression in CML is associated to imatinib treatment and to inhibition of leukemia cell proliferation. BMC Cancer 13:60. doi:10.1186/1471-2407-13-60

42. Pellagatti A, Cazzola M, Giagounidis A et al (2007) Expression profiling of CD34+ cells in patients with myelodysplastic syndromes: differences between early and advanced cases and analysis of apoptosis-related genes. Leuk Res 31:S35-S. doi:10.1016/ S0145-2126(07)70061-9

43. Alachkar H, Santhanam R, Maharry K et al (2014) SPARC promotes leukemic cell growth and predicts acute myeloid leukemia outcome. J Clin Invest 124:1512-1524. doi:10.1172/JCI70921

44. Powell JA, Thomas D, Barry EF et al (2009) Expression profiling of a hemopoietic cell survival transcriptome implicates osteopontin as a functional prognostic factor in AML. Blood 114:4859-4870. doi:10.1182/blood-2009-02-204818

45. Nakamura-Ishizu A, Okuno Y, Omatsu Y et al (2012) Extracellular matrix protein tenascin- $\mathrm{C}$ is required in the bone marrow microenvironment primed for hematopoietic regeneration. Blood 119:5429-5437. doi:10.1182/blood-2011-11-393645

46. Kim EK, Jeon I, Seo H et al (2014) Tumor-derived osteopontin suppresses antitumor immunity by promoting extramedullary myelopoiesis. Cancer Res 74:6705-6716. doi:10.1158/00085472.CAN-14-1482

47. Wang Z, Xiong S, Mao Y et al (2016) Periostin promotes immunosuppressive premetastatic niche formation to facilitate breast tumour metastasis. J Pathol 239:484-495. doi:10.1002/path.4747

48. Malanchi I, Santamaria-Martinez A, Susanto E, Peng H, Lehr HA, Delaloye JF, Huelsken J (2012) Interactions between cancer stem cells and their niche govern metastatic colonization. Cancer Res 72(Suppl):SY28-02. doi:10.1158/1538-7445. Am2012-Sy28-02

49. Sangaletti S, Tripodo C, Sandri S et al (2014) Osteopontin shapes immunosuppression in the metastatic niche. Cancer Res 74:4706-4719. doi:10.1158/0008-5472.CAN-13-3334

50. Condamine T, Dominguez GA, Youn JI, et al (2016) Lectin-type oxidized LDL receptor-1 distinguishes population of human polymorphonuclear myeloid-derived suppressor cells in cancer patients. Science Immunol 1:aaf8943. doi:10.1126/sciimmunol. aaf8943

51. Catena R, Bhattacharya N, El Rayes T et al (2013) Bone marrow-derived Gr1 + cells can generate a metastasis-resistant microenvironment via induced secretion of thrombospondin-1. Cancer Discov 3:578-589. doi:10.1158/2159-8290.CD-12.0476 